\title{
Effect of Base Sequence on G-Wire Formation in Solution
}

\author{
Lea Spindler, ${ }^{1,2}$ Martin Rigler, ${ }^{3}$ Irena Drevenšek-Olenik, ${ }^{2,3}$ Nason Ma'ani Hessari, ${ }^{4}$ \\ and Mateus Webba da Silva ${ }^{4}$
}

${ }^{1}$ Faculty of Mechanical Engineering, University of Maribor, Smetanova 17, 2000 Maribor, Slovenia

${ }^{2}$ J. Stefan Institute, Jamova 39, 1000 Ljubljana, Slovenia

${ }^{3}$ Faculty of Mathematics and Physics, University of Ljubljana, Jadranska 19, 1000 Ljubljana, Slovenia

${ }^{4}$ Faculty of Life and Health Sciences, University of Ulster, Cromore Road, Northern Ireland, Coleraine BT52 1SA, UK

Correspondence should be addressed to Lea Spindler, lea.spindler@uni-mb.si

Received 1 February 2010; Accepted 22 March 2010

Academic Editor: R. Eritja

Copyright ( 92010 Lea Spindler et al. This is an open access article distributed under the Creative Commons Attribution License, which permits unrestricted use, distribution, and reproduction in any medium, provided the original work is properly cited.

The formation and dimensions of G-wires by different short G-rich DNA sequences in solution were investigated by dynamic light scattering (DLS) and polyacrilamide gel electrophoresis (PAGE). To explore the basic principles of wire formation, we studied the effects of base sequence, method of preparation, temperature, and oligonucleotide concentration. Both DLS and PAGE show that thermal annealing induces much less macromolecular self-assembly than dialysis. The degree of assembly and consequently length of G-wires $(5-6 \mathrm{~nm})$ are well resolved by both methods for DNA sequences with intermediate length, while some discrepancies appear for the shortest and longest sequences. As expected, the longest DNA sequence gives the longest macromolecular aggregates with a length of about $11 \mathrm{~nm}$ as estimated by DLS. The quadruplex topologies show no concentration dependence in the investigated DNA concentration range $(0.1 \mathrm{mM}-0.4 \mathrm{mM})$ and no structural change upon heating.

\section{Introduction}

The construction of specific surface architectures via controlled self-assembly is a key goal in the design of nanoscale molecular electronic devices. In this respect, biomolecular self-assembly of the strongly interacting DNA bases provides a promising avenue. Particularly, attractive candidates are guanine $(\mathrm{G})$ and its derivatives, which are unique among the DNA constituents for their propensity to form quadruplex structures, known to be stabilized through Hoogsteen and Watson-Crick base pairing $[1,2]$. Moreover, guanine has a low ionization potential due to which it plays a key role in electrical conductivity of DNA-based materials.

Various research groups have recently reported on the evolution of the "art" of making surface deposited 1dimensional (1D) structures known as guanosine wires (Gwires) [3-12]. However, despite the promising experimental results, basic processes responsible for wire formation are still far from being fully understood. Two main steps are fundamental to drive the topic from a laboratory curiosity towards the technologically relevant G-wire engineering: (a) achieving control of self-folding and self-stimulated end-toend fusion of the macromolecular quadruplexes in solution as a function of their molecular composition (i.e., base sequence) and solution conditions, and (b) understanding the effect of surface interactions on material deposition from solution phase onto the solid substrate.

In this work, we report on how particular modifications of the base sequence of a G-rich oligonucleotide, which are supposed to affect the folding geometry of the quadruplex [13], affect its ability to form G-wires in solution phase. The idea of wire formation is based on the previously established finding that GC "sticky ends" may be used to link quadruplexes into longer 1D aggregates [14]. A series of oligonucleotide sequences with one or two GC ends and central sequences of different length were designed for this purpose (see Table 1). The folding topology [14, 15] has been previously investigated, and quadruplex formation has been recently studied by Mergny et al. [16]. In these studies, length and nature of propeller loops were studied, but end-to-end fusion of the quadruplexes was not considered. Here, we focused on end-to-end fusion of these quadruplex folds. 
Polyacrilamide gel electrophoresis (PAGE) is a conventional tool to differentiate between macromolecular objects of different length. During migration through the gel, breakage of bonds and smearing of bands may happen. Thus, weakly linked aggregates are difficult to be identified. An alternative noninvasive method for determining the size of macromolecular objects is dynamic light scattering (DLS). This method was extensively applied to study DNA molecules over a wide range of sizes [17-21]. Specifically, it has been used to characterize G-quartet stacking in solutions of single guanosine molecules [22-26], and for studying formation of G-quadruplexes [27-30]. Protozanova and Macgregor [29] compared the use of DLS and PAGE on sequences with long terminal tracks. These tracks were based on sequences such as $d\left(A_{15} G_{15}\right)$ and $d\left(A_{13} G_{15} T C\right)$, which form frayed wires with a $\mathrm{G}$-quadruplex stem and nonguanine portions reaching out as single-stranded arms. These frayed wires can be considerably long and have a rather broad size distribution. Thus, specific effects of base sequence on wire length were not evident.

We investigated the formation and dimensions of the supramolecular objects formed in aqueous solution of specially designed G-rich DNA sequences (Table 1) by combining DLS and PAGE measurements. We studied the effects of temperature, oligonucleotide concentration, method of preparation, and base sequence to explore the basic principles of G-wire formation.

\section{Experimental}

2.1. Material. Oligonucleotides (see Table 1) were ordered from Eurogentec (Belgium) as $40 \mathrm{nM}$ desalted syntheses and reconstituted in water. Oligonucleotides were folded utilising either dialysis or heat treatment. Dialysis was performed at a concentration of $100 \mu \mathrm{M}$ DNA in the presence of $100 \mathrm{mM} \mathrm{NaCl}$ buffered with $10 \mathrm{mM} \mathrm{NaPi}$ at $\mathrm{pH} 6.8$, then diluted in same concentration of buffer or $\mathrm{H}_{2} \mathrm{O}$ to specified concentrations immediately prior to measurements. Where heat treatment is specified, oligonucleotides at $100 \mu \mathrm{M}$ DNA in presence of $100 \mathrm{mM} \mathrm{NaCl}, 10 \mathrm{mM} \mathrm{NaPi}(\mathrm{pH}$ 6.8) were heated to $93^{\circ} \mathrm{C}$ for ten minutes and left to cool in heating block to room temperature prior to experiment. For DLS measurements, the oligonucleotides with a $0.4 \mathrm{mM}$ concentration were used. For the concentration dependence studies the initial solutions were dissolved by the corresponding buffer.

2.2. PAGE. Gel electrophoresis experiments were performed on 15\% native bis/acrylamide gels, utilising $1 \mathrm{X}$ TBE running buffer supplemented with $5 \mathrm{mM} \mathrm{NaBO}$ to retain folded architectures. Oligonucleotide samples were prepared to a final concentration of $2 \mu \mathrm{M}$ DNA in presence of $100 \mathrm{mM}$ $\mathrm{NaCl}$ buffered with $10 \mathrm{mM} \mathrm{NaPi}$ at $\mathrm{pH} 6.8$ with $5 \%$ sucrose to facilitate sample loading. Gels were run at $120 \mathrm{~V}$ and $4^{\circ} \mathrm{C}$ for a maximum of 2 hours and stained with $1 \mathrm{X}$ SYBR gold (Invitrogen, Paisley, UK) in 1X TBE buffer. In order to compare migration rates between different oligonucleotide
TABle 1: The investigated G-rich DNA sequences.

\begin{tabular}{ll}
\hline Designation & Sequence \\
\hline Sequence 1 & GCGGTCGGGGTCGGGGTCGG \\
Sequence 2 & GCGGAGGCG \\
Sequence 3 & GCGGTGGCG \\
Sequence 4 & GCGGTGGGGTGGCG \\
Sequence 5 & GCGGTGGGGTGGGGTGGGGTGGGGTGGCG \\
Sequence 6 & GCGGTGGGGTGGGGTGGCG
\end{tabular}

samples, a GeneRuler Ultra Low Range (10-200 bps) DNA Ladder (Fermentas, York, UK) was used.

2.3. Dynamic Light Scattering. Dynamic light scattering experiments (DLS) were performed using a digital correlator (ALV-Laser Vertriebgesellschaft), a goniometer, and a photomultiplier detector. The light source was a frequency doubled Nd:YAG laser with a wavelength of $532 \mathrm{~nm}$. The incident laser beam was linearly polarized in the direction perpendicular to the scattering plane. The scattered light of the same polarization was detected at scattering angles $50^{\circ} \leq \theta \leq 130^{\circ}$. The capillary containing the sample had an inner diameter of $5 \mathrm{~mm}$ and was immersed in an index matching bath with a diameter of $10 \mathrm{~cm}$ to minimize stray light from the outer capillary wall.

We measured the autocorrelation function $g_{2}(t)=$ $\langle I(0) I(t)\rangle /\langle I\rangle^{2}$ of the average scattered light intensity $I[31]$. Most of the measurements were performed in a mixed regime, in which the intensity autocorrelation function is related to the field correlation function $g_{1}(t)$ by the relation [32]

$$
g_{2}(t)=1+2\left(1-j_{d}\right) j_{d} g_{1}(t)+j_{d}^{2} g_{1}^{2}(t),
$$

with $j_{d}$ being the ratio between the intensity of the light that is scattered inelastically and the total scattered intensity. The field correlation function $g_{1}(t)$ for systems with a polydisperse size distribution can be expressed by stretch exponential functions [33]

$$
g_{1}(t)=\sum_{i} A_{i} \exp \left(\frac{-t}{\tau_{i}}\right)^{\beta_{i}},
$$

where $A_{i}$ is the amplitude of the $i$ th relaxation mode and the stretch exponent lies in the range $0 \leq \beta_{i} \leq 1$. The average relaxation time of the $i$ th relaxation mode is given by

$$
\left\langle\tau_{i}\right\rangle=\frac{\tau_{i}}{\beta_{i}} \Gamma\left(\frac{1}{\beta_{i}}\right),
$$

where $\Gamma\left(1 / \beta_{i}\right)$ is the gamma function. The parameter $\beta_{i}$ is a measure of the width of the distribution of relaxation times. Very narrow distributions correspond to $\beta_{i} \sim 1$, while smaller values of $\beta_{i}$ indicate broader distributions. The characteristic relaxation times of the observed dynamic modes were obtained by fitting the measured autocorrelation curves $g_{2}(t)$ to (1) and (2). 


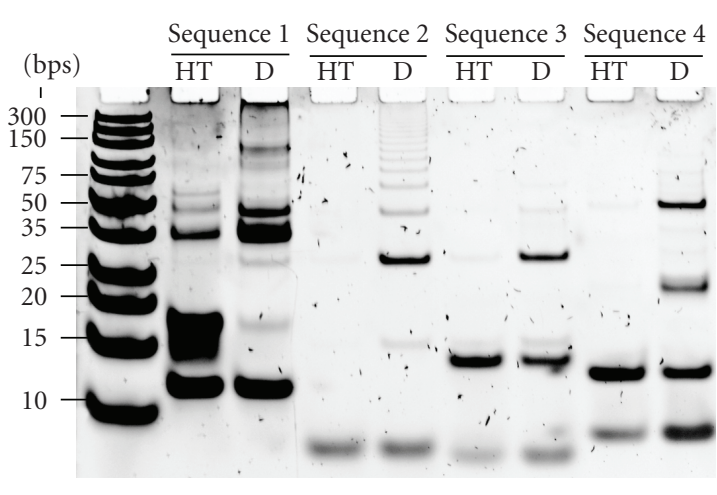

(a)

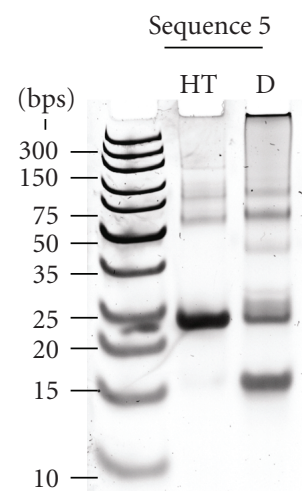

(b)

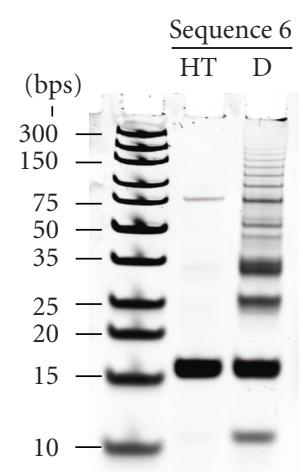

(c)

FIgURE 1: PAGE shows that heat treatment (HT) mainly gives short aggregates, while preparation by dialysis (D) results in higher degree of assembly. Most profound multistep assembling is observed for dialysed sequences 1,5 , and 6 .

According to the polarized scattering (so called VV scattering) detected in our experiments, the translational diffusion coefficients were calculated as

$$
D_{i}=\frac{1}{\left\langle\tau_{i}\right\rangle q^{2}}
$$

where $q$ is the scattering wave vector given as $q=$ $(4 \pi n / \lambda) \sin (\theta / 2)$ with $n=1.33$ being the solution refractive index, $\lambda$ the laser wavelength and $\theta$ the scattering angle. In most cases, two diffusive modes were detected. Several correlation curves were measured for every solution and averaged values of the fitting parameters were taken for further consideration.

From the translational diffusion coefficients, the dimensions of the scattering objects in solutions can be estimated. The corresponding hydrodynamic radius is calculated as

$$
r_{H}=\frac{k_{B} T}{6 \pi \eta D}
$$

with $k_{B}$ being the Boltzmann constant, $T$ the absolute temperature, and $\eta$ the solvent viscosity. For dilute solutions of rod-like particles the hydrodynamic theory of Tirado and Garcia de la Torre [34-36] can be applied as long as the ratio of length to diameter $p=L / d$ is in the range $2 \leq p \leq 30$. The translational diffusion coefficient in this theory is given by

$$
D=\frac{k_{B} T}{3 \pi \eta L}(\ln p+\nu)
$$

where $v$ is the end-effect correction term given as $v=0.312+$ $0.565 / p-0.100 / p^{2}$. Knowing the diameter of the studied rodlike assembly, thus the length of the objects can be estimated.

\section{Results}

3.1. PAGE. The sequences were first investigated by PAGE to check for folding of oligonucleotides into higher order architectures. Samples prepared by heat treatment show a lesser degree of macromolecular assembly as compared to dialysed samples for all investigated oligonucleotides (Figure 1). The

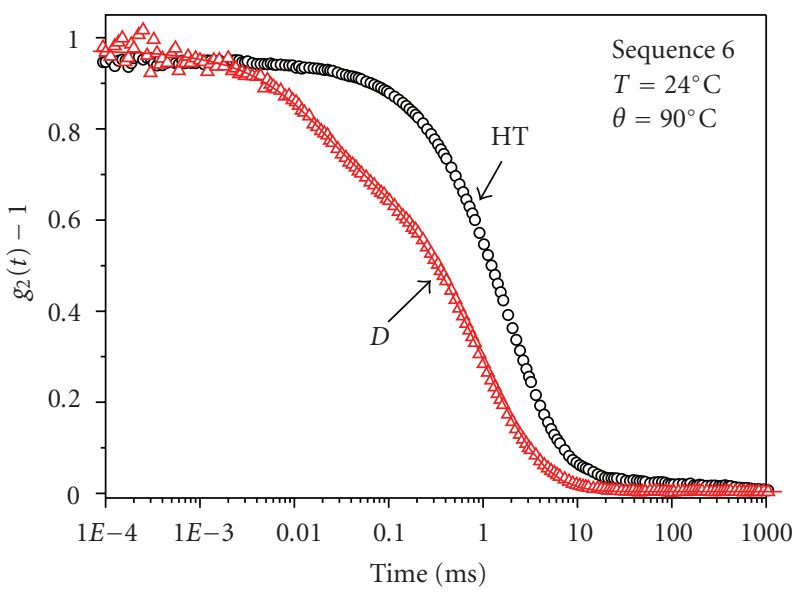

Figure 2: Typical DLS autocorrelation functions detected for heat treated (HT) and dialysed (D) samples. The slow diffusive mode (correlation time in the range of $1-10 \mathrm{~ms}$ at $\theta=90^{\circ}$ ) is observed for both samples, while the fast diffusive mode (correlation time in the range of $0.01-0.1 \mathrm{~ms}$ at $\theta=90^{\circ}$ ) is observed only for the dialysed sample. The fast mode is assigned to quadruplex motion, while the slow mode presumably arises from dynamics of loose polyion associates.

formation of macromolecular assemblies should indeed take more time. The band with the highest mobility in each lane corresponds to a bistranded monomeric unit. The shortest dialysed sequences (sequences 2 and 3 ) show only few albeit sharp bands pointing to the formation of smaller discrete stable species and a very low degree of macromolecular selfassembly. The longer sequences 1, 5 and 6, on the other hand, indicate possible stepwise assembly into large aggregates.

3.2. DLS. In all investigated samples, we detected a slow diffusive mode with correlation times in the range of 1-10 ms (at $\theta=90^{\circ}$ ) (Figures 2 and 3). Such a mode corresponds to translational motion of large globular aggregates with hydrodynamic radii in the range of micrometers. Similar slow modes are typically observed in DNA and many other 


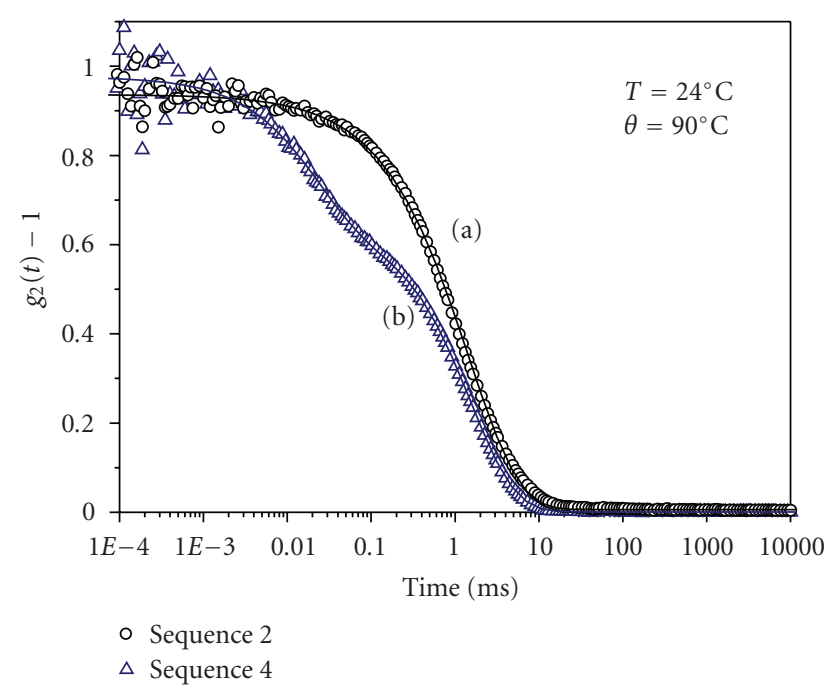

FIgURE 3: Typical autocorrelation functions of short (a) and long (b) oligonucleotide solutions prepared by dialysis method ( $c=$ $0.4 \mathrm{mM})$.

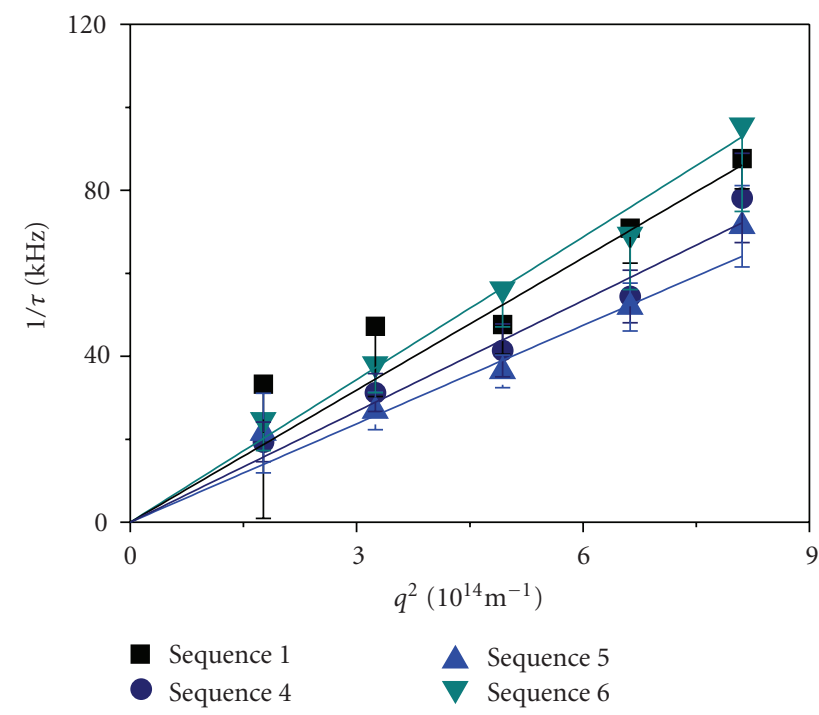

Figure 4: Dispersion relation of the fast diffusive mode for dialysed solutions of sequences $1,4,5$, and 6 at $T=24^{\circ} \mathrm{C}$. Solid lines are fits to (4). Error bars were obtained by fitting experimental correlation functions $g_{2}(t)$ to (1) taking into account (2).

polyelectrolyte solutions. They are usually attributed to the presence of loose multichain associates formed due to electrostatic interactions, but their nature is still not quite resolved $[20,37,38]$. As these aggregates are to our opinion not connected with G-wire formation, they will not be further considered.

In all heat treated samples, only the above mentioned "slow mode" exists, so they will not be further discussed. Solely the "slow mode" is observed also in the dialysed sequence 2 (Figure 3(a)), which is the only sequence containing adenine base. In dialysed sequence 3 , an additional fast diffusive mode can be faintly resolved at large
TABLE 2: Hydrodynamic parameters obtained from DLS measurements in dialysed samples at $0.4 \mathrm{mM}$ DNA concentration and room temperature. The length of the quadruplex $L$ is estimated from (6) using $d=2.6 \mathrm{~nm}$ for the quadruplex diameter [26, 39]. For comparison, the length of extended oligonucleotides $L_{\text {oligo }}$ is estimated by multiplying the number of bases with $0.34 \mathrm{~nm}$, the average base distance.

\begin{tabular}{lcccc}
\hline Designation & $\begin{array}{c}D_{f} \\
\left(10^{10} \mathrm{~m}^{2} / \mathrm{s}\right)\end{array}$ & $r_{H}(\mathrm{~nm})$ & $\begin{array}{c}\text { Length } \\
(\mathrm{nm})\end{array}$ & $\begin{array}{c}L_{\text {oligo }} \\
(\mathrm{nm})\end{array}$ \\
\hline Sequence 1 & $1.06 \pm 0.037$ & $2.26 \pm 0.034$ & $6.1 \pm 0.5$ & 6.8 \\
Sequence 2 & - & - & - & 3.1 \\
Sequence 3 & $3.0 \pm 0.30$ & $0.78 \pm 0.08$ & - & 3.1 \\
Sequence 4 & $0.89 \pm 0.040$ & $2.69 \pm 0.044$ & $8.8 \pm 0.8$ & 4.8 \\
Sequence 5 & $0.79 \pm 0.008$ & $3.03 \pm 0.062$ & $11.0 \pm 0.2$ & 9.9 \\
Sequence 6 & $1.15 \pm 0.041$ & $2.09 \pm 0.036$ & $5.0 \pm 0.4$ & 6.5 \\
\hline
\end{tabular}

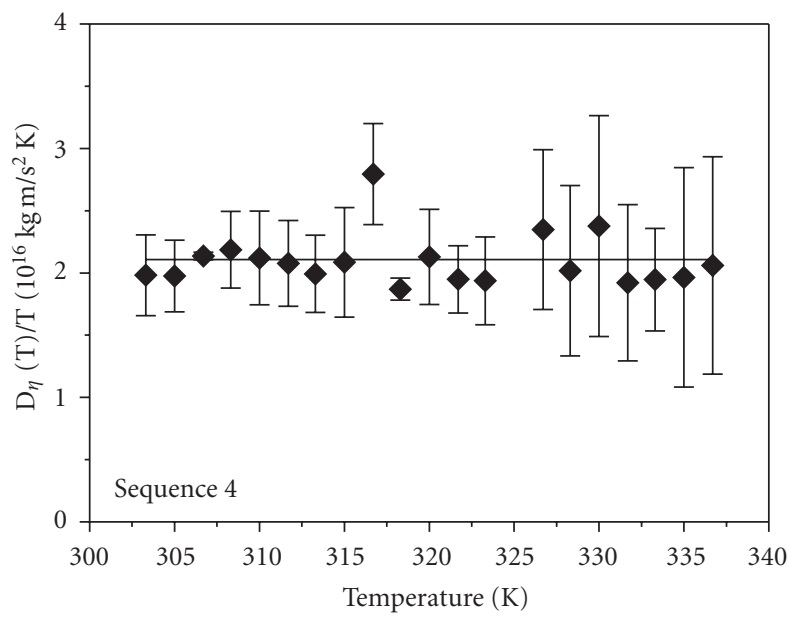

Figure 5: The values of the diffusion coefficient of the fast mode $D_{f}$ normalised by solution temperature $T$ and solvent viscosity $\eta(T)$ show no modifications with temperature. This suggests that the associated quadruplex structure is stable in the whole investigated temperature range.

scattering angles $\left(\theta>90^{\circ}\right)$. The corresponding diffusion coefficient calculated from (4) has a value of $D_{f}=3.0 \pm$ $0.3 \cdot 10^{-10} \mathrm{~m}^{2} / \mathrm{s}$ (Table 2), which indicates fast translational motion of very small scattering objects, most probably single oligonucleotides. In dialysed sequence 4 , the fast mode is more profound and can be clearly resolved (Figure 3(b)).

The DLS autocorrelation functions of dialysed sequences containing GGGG repeats (sequences 1, 5, and 6) all show two relaxation modes. The angular dependence of the inverse relaxation time of the fast mode (Figure 4) reveals quadratic dispersion as given by (4). The resultant diffusion coefficients $D_{f}$ obtained for solution concentration of $0.4 \mathrm{mM}$ are listed in Table 2. The obtained values suggest that the fast mode is most probably associated with translational motion of oligonucleotides assembled into G-quadruplex structures.

The dialysed sequence 4 at $0.4 \mathrm{mM}$ DNA was also used to investigate the temperature dependence of the fast diffusive 


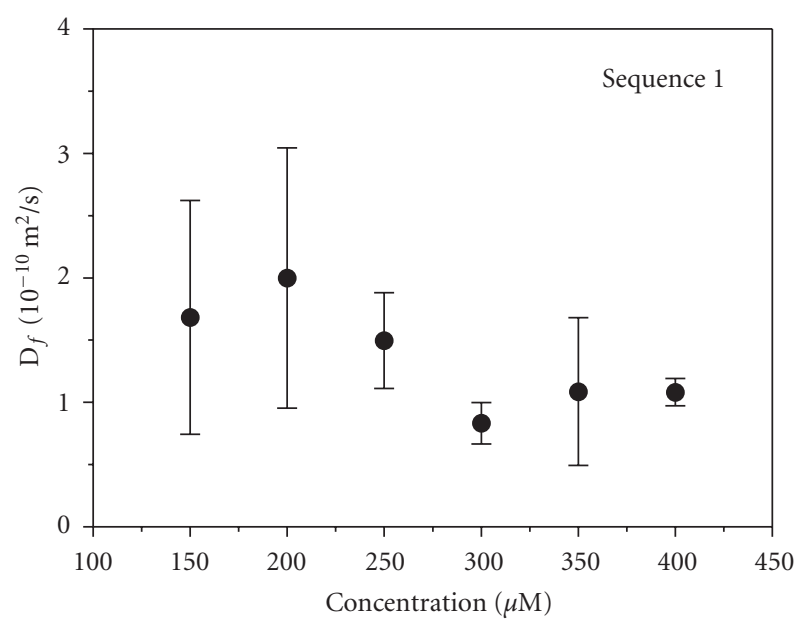

FIGURE 6: Diffusion coefficient of the fast diffusive mode as a function of oligonucleotide concentration. Measurements for dialysed sequence 1 .

mode (Figure 5). The sample was slowly heated from $T=$ $300 \mathrm{~K}$ towards higher temperatures. A relative amplitude of the fast mode decreased with increasing temperature and for $T>338 \mathrm{~K}$ the fast mode could not be resolved anymore. However, the slow mode remained. To reveal possible temperature-induced structural changes of the scattering objects, the values of diffusion coefficient $D_{f}$ obtained at different temperatures were divided by temperature $T$ and multiplied by the corresponding solvent viscosity $\eta(T)$. The resulting values of $D_{f} \eta(T) / T$ as a function of temperature are shown in Figure 5. One can notice that they remain constant throughout the investigated temperature range, which indicates that no structural modification of the scattering objects (quadruplexes) takes place.

The effect of oligonucleotide concentration on the value of $D_{f}$ was investigated for dialyzed sequence 1 (Figure 6). Although it appears that the values decrease with increasing concentration, no precise conclusion can be drawn due to the large uncertainty of the measured data points. At low solution concentrations and short relaxation times, DLS measurements typically exhibit relatively large noise level. This has been previously observed in studies on short DNA sequences [19]. However, for DNA concentrations below $1 \mathrm{mM}$, which was the case in all our measurements, the effect of electrostatic interactions on solution dynamics is usually negligible and so the solution can be considered as infinitely dilute. Consequently, the value of the diffusion coefficient is expected to be constant.

\section{Discussion}

Both, PAGE and DLS analysis, reveal that the method of sample preparation is essential for the level of self-assembly in solution. We observe formation of slower migrating species for all oligonucleotides in PAGE for the dialysis treated samples. Sequences 1, 2, 5, and 6 appear to form larger assemblies, whilst sequences 3 and 4 do not. The most striking results are for sequences 2 and 6, where one or two species of lower molecular weight were formed in the annealing treatment versus numerous larger species through dialysis.

PAGE comparison between sequences 2 and 3 shows that there are sequence effects in the kinetics of formation for the same topology. When comparing dialysis, a band appearing at nominal $15 \mathrm{bp}$ in sequence 3 appears only very faintly in sequence 2, while a faster, and a slower, migrating bands appear quite distinctively in both. The effect of "sticky ends" is also shown when comparing sequences 1 and 6 . While between nominal 10 and $35 \mathrm{bp}$ they have the same number of bands appearing in approximately the same positions, their intensities vary substantially. For these particular sequences, the difference in the annealing treatment is greater, with sequence 6 showing a very strong fast migrating band at nominal $16 \mathrm{bp}$. In contrast, the fastest migrating band for seq 1 is at nominal $12 \mathrm{bp}$. In general, and as expected, it is apparent that the greater the oligonucleotide length the longer the species formed. Indeed, it is apparent for the longest oligonucleotide sequence (sequence 5) that the macromolecular DNA objects break into smaller units in a less discrete manner than for the other sequences. The smear for low migrating DNA in sequence 5 may thus be due to steric hindrance by the pore size of the gel.

For the shortest of the dialysed sequences, sequences 2 and 3 , the absence of any measurable fast DLS mode for the former and the observation of a faint extremely fast mode for the latter, can be explained only by the presence of very small scattering objects, most probably single oligonucleotide molecules. This finding is not consistent with PAGE results (Figure 1), which reveal additional slower diffusing species resulting in sharp bands, probably signifying multimers. Similar discrete bands are seen also in the picture of PAGE for dialysed sequence 4. For this sequence, the PAGE result is in better agreement with the DLS results. The diffusion coefficient of the fast DLS mode gives quadruplex length of $8.8 \pm 0.8 \mathrm{~nm}$ (Table 2), which is considerably larger than the length of a single oligonucleotide strand (about $4.8 \mathrm{~nm}$ ). This signifies formation of assemblies formed from several oligonucleotides.

The two dialysed intermediate-length sequences (sequences 1 and 6) both exhibit the same PAGE band attributed to fast migrating monomers and a strong band at about $35 \mathrm{bps}$ (accordingly to the reference duplex DNA ladder). The main difference between the two oligonucleotides is in the formation of two intermediate bands for sequence 6 , while sequence 1 forms no intermediate species. Interestingly, the two strong bands (at $35 \mathrm{bps})$ very well agree with the quadruplex length $L$ estimated from the DLS measurements, which is $5.0 \pm$ $0.4 \mathrm{~nm}$ for sequence 6 and $6.1 \pm 0.5 \mathrm{~nm}$ for sequence 1 , respectively. The smaller apparent length of sequence 6 can be explained by the presence of the intermediate species contributing to the scattering. The diffusion coefficient measured for sequence $1\left(D_{f}=1.06 \pm 0.037\right)$ is in very good agreement with the values obtained for similar folded structures by hydrodynamic modelling [30]. 
Journal of Nucleic Acids

The longest oligonucleotide, sequence 5, forms the largest aggregates. In PAGE, the majority of the material is so slowly migrating through the gel, that just a broad smearing is observed. Therefore, a formation of very long structures is expected. DLS measurements, on the other hand, reveal the presence of supramolecular assemblies with the length of $11.0 \pm 0.2 \mathrm{~nm}$, which is only slightly larger than the length of a single strand. In contrast to PAGE, DLS also reveals a relatively narrow distribution of aggregate lengths. This is seen from the DLS stretch exponent factor for the fast mode, which is in the range $0.9<\beta_{f}<1$, thus excluding a broad distribution of the dimensions of the scattering objects. Also for other sequences, the values of $\beta_{f}$ were very close to 1 .

The origin of the differences between the PAGE and the DLS results is not clear. On the basis of the PAGE results, sequences 1,5 , and 6 are all supposed to form some long wire-like assemblies, but DLS measurements do not support this expectations. Accordingly to the DLS results, the highest level of self-assembly is expected for sequence 4, which exhibits the largest length of the scattering objects with respect to the length of the single strand. One possible reason for the discrepancies might be quite different solution concentrations used for the two experiments. PAGE is usually performed with concentrations $c \sim 10 \mu \mathrm{M}$, while DLS gives reasonable signals. Yet for $c>100 \mu \mathrm{M}$, this is not consistent with what we intuitively know, that is, higher concentrations tend to favour multimer formation. Another aspect arises from the slow DLS mode, which signifies that in addition to wire-like assemblies there are also some other aggregate types present in solution. These aggregates, most probably loose multipolyion associates, might correspond to the slow diffusing objects observed in PAGE.

DLS experiments bring also information on temperature and concentration dependence of the self-assembly. The temperature dependence (Figure 5) shows that up to $T=$ $65^{\circ} \mathrm{C}$ the length of the self-assembled objects remains constant. Nevertheless, the amplitude of the fast DLS mode strongly decreases by increasing temperature, while the overall scattering intensity is only slightly reduced. This signifies that the slow mode, which mainly contributes to the scattering intensity, is not much affected by heating. But relatively to it, the scattering intensity related to the fast mode becomes more and more weak. This can be explained by temperature-driven dissociation of the selfassembled structures of well-defined size into much smaller single oligonucleotides, which are practically invisible by DLS.

Dependence of the fast DLS mode on the concentration of the solution (Figure 6) also supports the idea of formation of the self-assembled structures with welldefined length, which are not affected by modifications of the solution concentration. On the contrary to wire-like assemblies formed from single guanosine molecules (GMP), it seems that formation of supramolecular assemblies of Grich oligonucleotides, does not exhibit any critical solution concentration, at which the assembling would become profound.

\section{Conclusions}

A series of G-rich oligonucleotides was studied by PAGE and DLS to investigate the formation and dimensions of Gquadruplexes. Both methods show that thermal annealing induces much less macromolecular self-assembly than the dialysis method. This demonstrates that not only the base sequence, but also the folding kinetics play an important role in the self-assembly process. On one hand, this makes the phenomenon very complex, but on the other hand it provides a possibility for fine tuning of the assembling features via external stimuli. Further studies are needed to find the source of the differences and how they can be modulated.

PAGE and DLS show the best agreement on quadruplex dimensions for sequences of intermediate length (sequences 1, 4 and 6). The last band of sequence 4 coincides with the DLS signal arising from aggregates of a $9 \mathrm{~nm}$ length. The two similar sequences 1 and 6 give also aggregates of similar length $5-6 \mathrm{~nm}$ and these agree with a strong PAGE band observed for both sequences.

For the shortest sequences 2 and 3, PAGE suggest the formation of multimers not detected by DLS. The only DLS signal comes from very fast diffusing objects with effective dimensions below $1 \mathrm{~nm}$ pointing to single oligonucleotides. For the largest sequence, on the other hand, PAGE suggests very long aggregates giving a broad smeared band at the beginning of the lane. In DLS instead, a well defined fast mode is attributed to a species with the length of about $11 \mathrm{~nm}$.

Because we are able to assess intermediate lengths, we are currently investigating the mechanism of self-assembly of these wires by combining both methods. Thus, the combination provides valuable information on the G-quadruplex formation towards control of its length.

\section{Acknowledgment}

The authors acknowledge the financial support of COST Action MP0802 in scope of Short Term Scientific Missions.

\section{References}

[1] J. R. Williamson, "G-quartet structures in telomeric DNA," Annual Review of Biophysics and Biomolecular Structure, vol. 23, pp. 703-730, 1994.

[2] J. T. Davis, "G-quartets 40 years later: from 5'-GMP to molecular biology and supramolecular chemistry," Angewandte Chemie International Edition, vol. 43, no. 6, pp. 668-698, 2004.

[3] T. C. Marsh and E. Henderson, "G-wires: self-assembly of a telomeric oligonucleotide, d(GGGGTTGGGG), into large superstructures," Biochemistry, vol. 33, no. 35, pp. 1071810724, 1994.

[4] M. Weglarz, J. Vesenka, W. Fritzsche, S. Yerkes, and E. Kmiec, "Analysis of G-5x Quadruplex DNA," in International Symposium on DNA-Based Nanodevices, vol. 1062 of AIP Conference Proceedings, pp. 123-128, 2008.

[5] J. Vesenka, D. Bagg, A. Wolff, A. Reichert, R. Moeller, and W. Fritzsche, "Auto-orientation of G-wire DNA on mica," Colloids and Surfaces B, vol. 58, no. 2, pp. 256-263, 2007. 
[6] K. Kunstelj, F. Federiconi, L. Spindler, and I. DrevenšekOlenik, "Self-organization of guanosine 5'-monophosphate on mica," Colloids and Surfaces B, vol. 59, no. 2, pp. 120-127, 2007.

[7] M. A. Batalia, E. Protozanova, R. B. Macgregor Jr., and D. A. Erie, "Self-assembly of frayed wires and frayed-wire networks: nanoconstruction with multistranded DNA," Nano Letters, vol. 2, no. 4, pp. 269-274, 2002.

[8] T. C. Marsh, J. Vesenka, and E. Henderson, "A new DNA nanostructure, the G-wire, imaged by scanning probe microscopy," Nucleic Acids Research, vol. 23, no. 4, pp. 696700, 1995.

[9] N. Borovok, N. Iram, D. Zikich, et al., "Assembling of Gstrands into novel tetra-molecular parallel G4-DNA nanostructures using avidin-biotin recognition," Nucleic Acids Research, vol. 36, no. 15, pp. 5050-5060, 2008.

[10] I. Lubitz, N. Borovok, and A. Kotlyar, "Interaction of monomolecular G4-DNA nanowires with TMPyP: evidence for intercalation," Biochemistry, vol. 46, no. 45, pp. 1292512929, 2007.

[11] K. Kunstelj, L. Spindler, F. Federiconi, M. Bonn, I. DrevenšekOlenik, and M. Čopič, "Sum-frequency generation spectroscopy of self-assembled structures of Guanosine $5^{\prime}$ monophosphate on mica," Chemical Physics Letters, vol. 467, no. 1-3, pp. 159-163, 2008.

[12] J. B. Hightower, D. R. Olmos, and J. A. Walmsley, "Supramolecular structure and polymorphism of alkali metal salts of guanosine $5^{\prime}$-monophosphate: SEM and NMR study," Journal of Physical Chemistry B, vol. 113, no. 36, pp. 1221412219, 2009.

[13] M. Webba da Silva, "Geometric formalism for DNA quadruplex folding," Chemistry, vol. 13, no. 35, pp. 9738-9745, 2007.

[14] M. Webba da Silva, "Association of DNA Quadruplexes through G:C:G:C tetrads. Solution structure of d(GCGGTGGAT)," Biochemistry, vol. 42, no. 49, pp. 1435614365, 2003.

[15] M. Webba da Silva, "Experimental demonstration of T:(G:G:G:G):T hexad and T:A:A:T tetrad alignments within a DNA quadruplex stem," Biochemistry, vol. 44, no. 10, pp. 3754-3764, 2005.

[16] J.-L. Mergny, A. De Cian, S. Amrane, and M. Webba da Silva, "Kinetics of double-chain reversals bridging contiguous quartets in tetramolecular quadruplexes," Nucleic Acids Research, vol. 34, no. 8, pp. 2386-2397, 2006.

[17] J. Seils and R. Pecora, "Dynamics of a 2311 base pair superhelical DNA in dilute and semidilute solutions," Macromolecules, vol. 28, no. 3, pp. 661-673, 1995.

[18] H. Tj. Goinga and R. Pecora, "Dynamics of low molecular weight DNA fragments in dilute and semidilute solutions," Macromolecules, vol. 24, no. 23, pp. 6128-6138, 1991.

[19] H. Liu, L. Skibinska, J. Gapinski, A. Patkowski, E. W. Fischer, and R. Pecora, "Effect of electrostatic interactions on the structure and dynamics of a model polyelectrolyte. I. Diffusion," Journal of Chemical Physics, vol. 109, no. 17, pp. 7556-7566, 1998.

[20] L. Skibinska, J. Gapinski, H. Liu, A. Patkowski, E. W. Fischer, and R. Pecora, "Effect of electrostatic interactions on the structure and dynamics of a model polyelectrolyte. II. Intermolecular correlations," Journal of Chemical Physics, vol. 110, no. 3, pp. 1794-1800, 1999.

[21] A. Wilk, J. Gapinski, A. Patkowski, and R. Pecora, "Selfdiffusion in solutions of a 20 base pair oligonucleotide: effects of concentration and ionic strength," Journal of Chemical Physics, vol. 121, no. 21, pp. 10794-10802, 2004.
[22] W. Eimer and Th. Dorfmüller, "Self-aggregation of guanosine $5^{\prime}$-monophosphate studied by dynamic light scattering techniques," Journal of Physical Chemistry, vol. 96, no. 16, pp. 6790-6800, 1992.

[23] L. Spindler, I. Drevenšek-Olenik, M. Čopič, et al., "Dynamic light scattering and ${ }^{31} \mathrm{P}$ NMR spectroscopy study of the selfassembly of deoxyguanosine 5'-monophosphate," European Physical Journal E, vol. 7, no. 1, pp. 95-102, 2002.

[24] L. Spindler, I. Drevenšek-Olenik, M. Čopič, J. Cerar, J. Škerjanc, and P. Mariani, "Dynamic light scattering and ${ }^{31} \mathrm{P}$ NMR study of the self-assembly of deoxyguanosine $5^{\prime}$ monophosphate: the effect of added salt," European Physical Journal E, vol. 13, no. 1, pp. 27-33, 2004.

[25] H. Jurga-Nowak, E. Banachowicz, A. Dobek, and A. Patkowski, "Supramolecular guanosine 5'-monophosphate structures in solution. Light scattering study," Journal of Physical Chemistry B, vol. 108, no. 8, pp. 2744-2750, 2004.

[26] A. Wong, R. Ida, L. Spindler, and G. Wu, "Disodium guanosine $5^{\prime}$-monophosphate self-associates into nanoscale cylinders at $\mathrm{pH}$ 8: a combined diffusion NMR spectroscopy and dynamic light scattering study," Journal of the American Chemical Society, vol. 127, no. 19, pp. 6990-6998, 2005.

[27] M. Bolten, M. Niermann, and W. Eimer, "Structural analysis of G-DNA in solution: a combination of polarized and depolarized dynamic light scattering with hydrodynamic model calculations," Biochemistry, vol. 38, no. 38, pp. 12416-12423, 1999.

[28] A. Włodarczyk, J. Gapiński, A. Patkowski, and A. Dobek, "Structural polymorphism of telomeres studied by photon correlation spectroscopy," Acta Biochimica Polonica, vol. 46, no. 3, pp. 609-613, 1999.

[29] E. Protozanova and R. B. Macgregor Jr., "Thermal activation of DNA frayed wire formation," Biophysical Chemistry, vol. 84, no. 2, pp. 137-147, 2000.

[30] A. Włodarczyk, P. Grzybowski, A. Patkowski, and A. Dobek, "Effect of ions on the polymorphism, effective charge, and stability of human telomeric DNA. Photon correlation spectroscopy and circular dichroism studies," Journal of Physical Chemistry B, vol. 109, no. 8, pp. 3594-3605, 2005.

[31] B. J. Berne and R. Pecora, Dynamic Light Scattering, Wiley, New York, NY, USA, 1976.

[32] A. Mertelj, L. Cmok, and M. Čopič, "Anomalous diffusion in ferrofluids," Physical Review E, vol. 79, no. 4, Article ID 041402, 2009.

[33] K. S. Schmitz, An Introduction to Dynamic Light Scattering by Macromolecules, Academic Press, San Diego, Calif, USA, 1990.

[34] M. M. Tirado and J. Garcia de la Torre, "Translational friction coefficients of rigid, symmetric top macromolecules. Application to circular cylinders," The Journal of Chemical Physics, vol. 71, no. 6, pp. 2581-2587, 1979.

[35] M. M. Tirado and J. Garcia de la Torre, "Rotational dynamics of rigid, symmetric top macromolecules. Application to circular cylinders," The Journal of Chemical Physics, vol. 73, no. 4, pp. 1986-1993, 1980.

[36] M. M. Tirado, C. Lopez Martinez, and J. Garcia de la Torre, "Comparison of theories for the translational and rotational diffusion coefficients of rod-like macromolecules. Application to short DNA fragments," The Journal of Chemical Physics, vol. 81, no. 4, pp. 2047-2052, 1984.

[37] M. Sedlak, "Long-time stability of multimacroion domains in polyelectrolyte solutions," Journal of Chemical Physics, vol. 116, no. 12, pp. 5246-5255, 2002. 
[38] M. Sedlak, "Mechanical properties and stability of multimacroion domains in polyelectrolyte solutions," Journal of Chemical Physics, vol. 116, no. 12, pp. 5236-5245, 2002.

[39] M. S. Kaucher, W. A. Harrell Jr., and J. T. Davis, "A unimolecular G-quadruplex that functions as a synthetic transmembrane $\mathrm{Na}^{+}$transporter," Journal of the American Chemical Society, vol. 128, no. 1, pp. 38-39, 2006. 

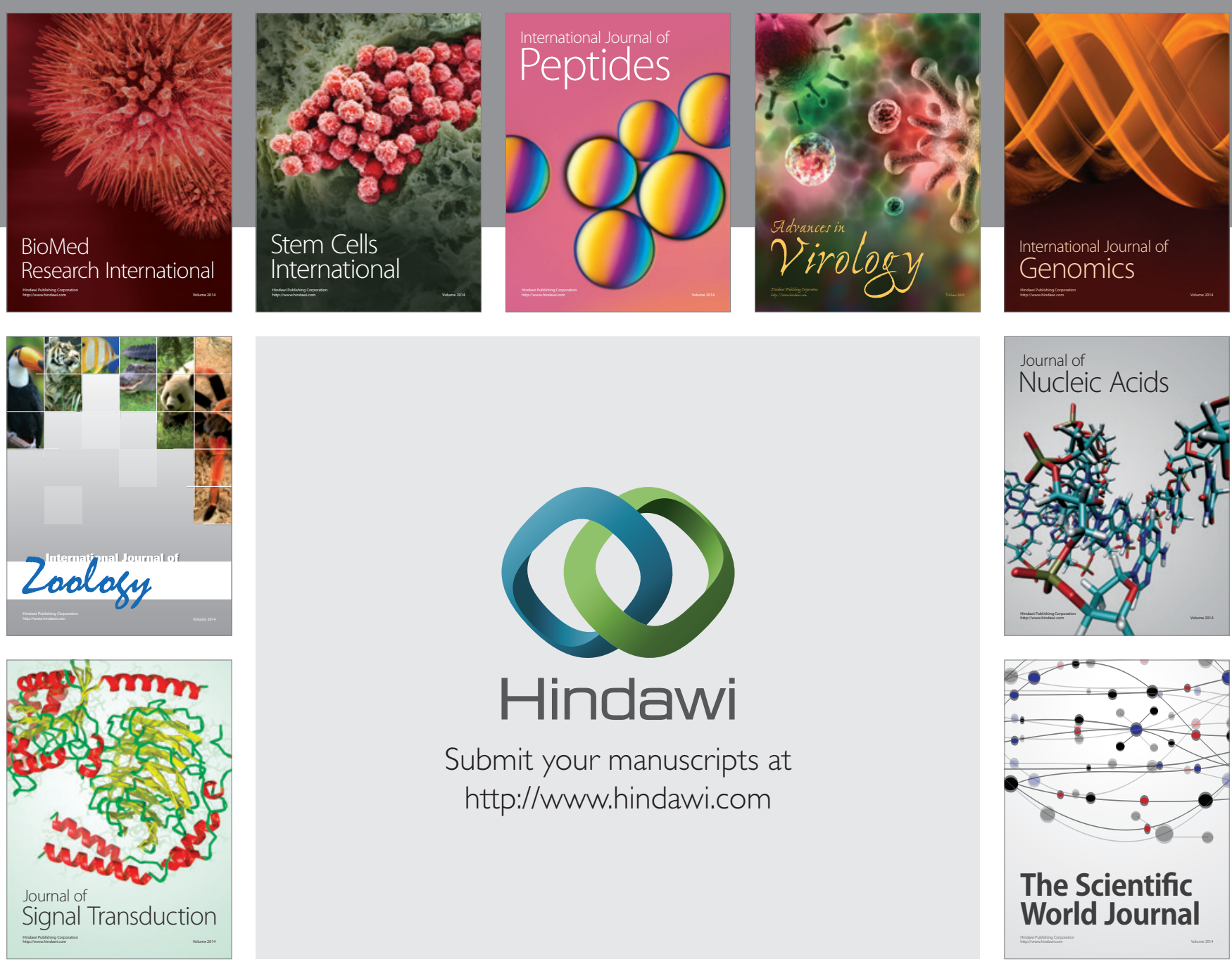

Submit your manuscripts at

http://www.hindawi.com
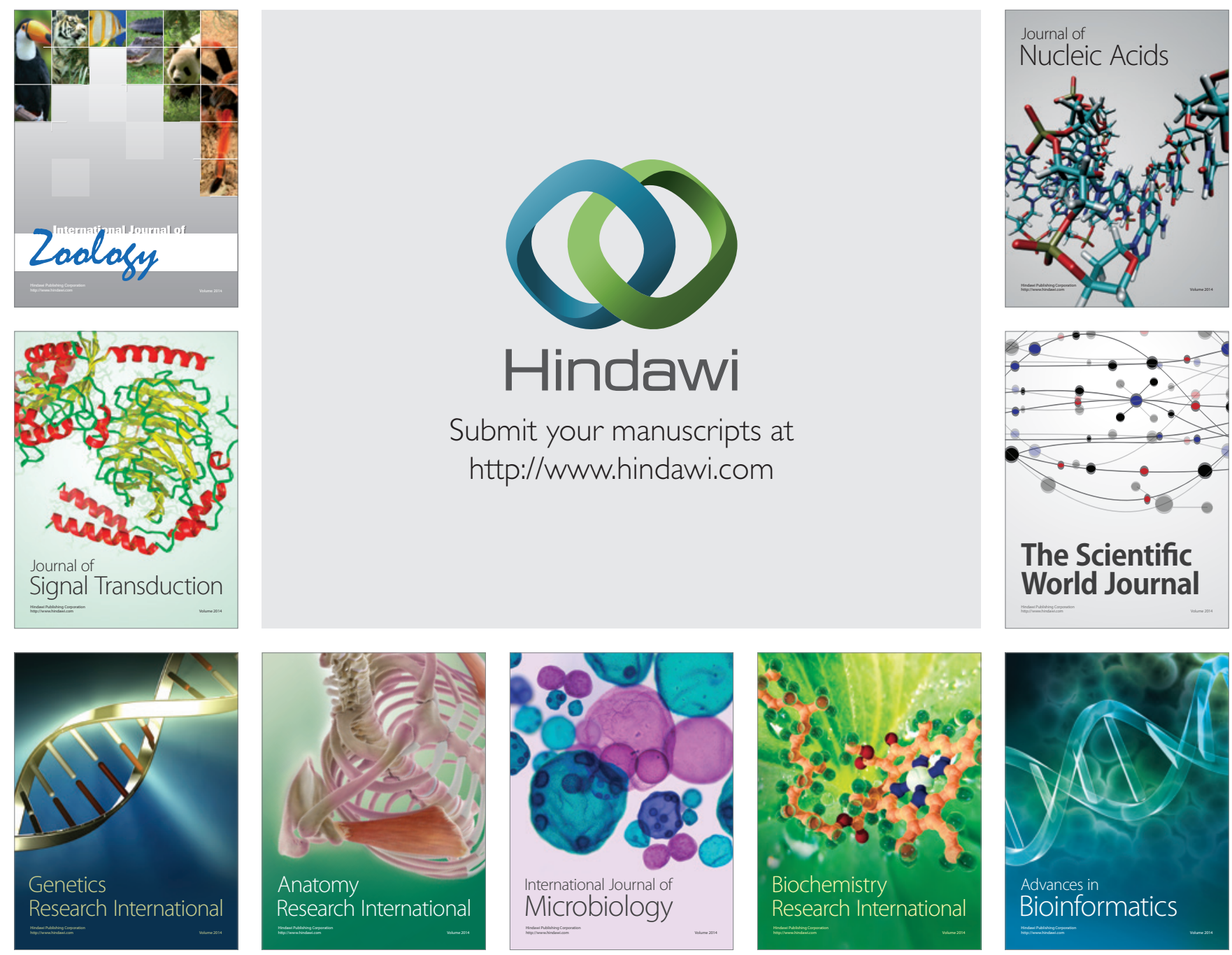

The Scientific World Journal
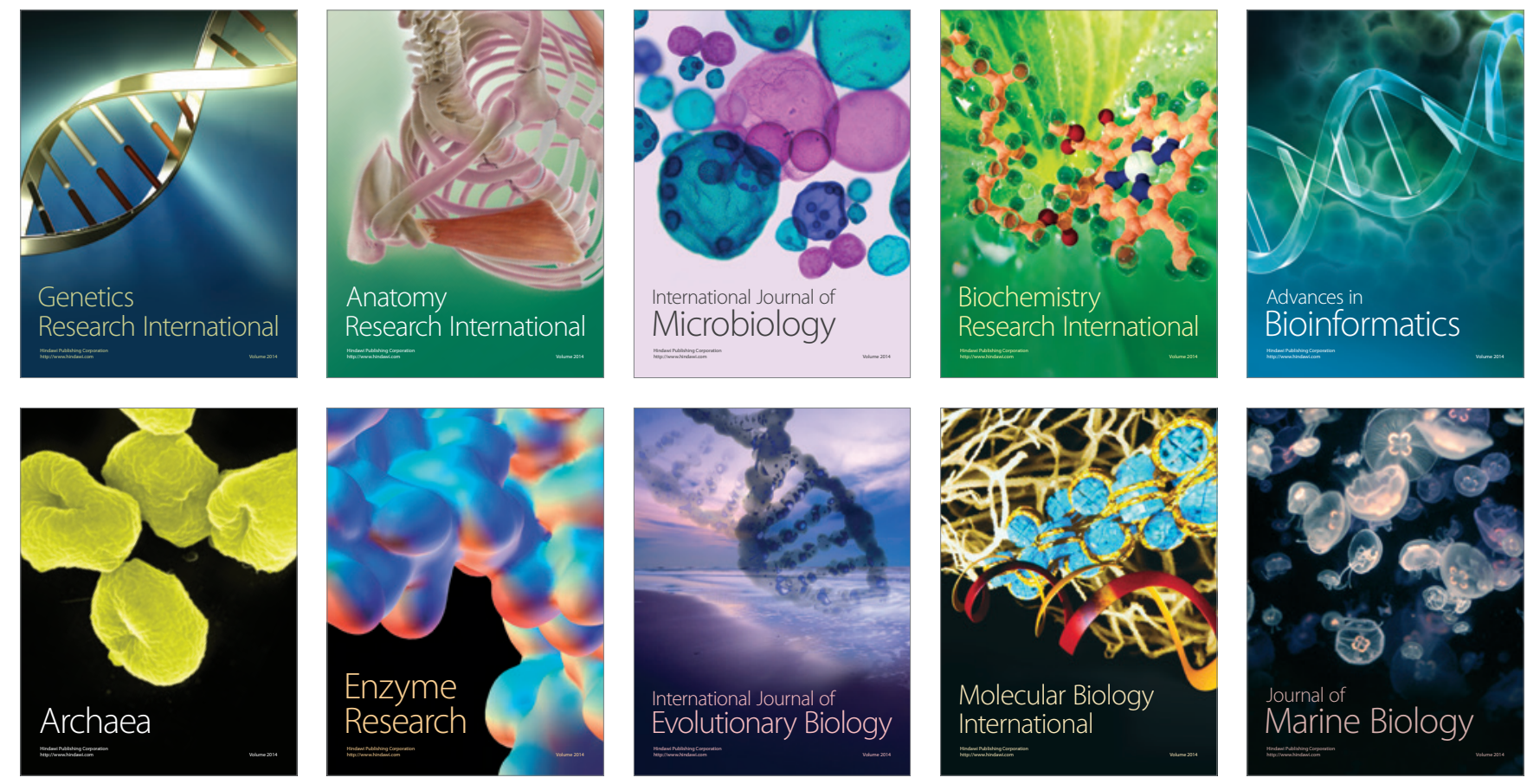